\title{
Glycerophospholipids in sea cucumber (Stichopus japonicus) and its processing by-products serve as bioactives and functional food ingredients
}

\author{
Xin Zhou ${ }^{\mathrm{a}}$, Da-Yong Zhou ${ }^{\mathrm{a}, \mathrm{b}^{*}}$, Fa-Wen Yin ${ }^{\mathrm{a}}$, Liang Songa,b, Yu-Xin Liu ${ }^{\mathrm{a}}$, Hong-Kai Xie ${ }^{\mathrm{c}}$, \\ Kai-Qi Ganga ${ }^{\mathrm{a}}$, Bei-Wei Zhu ${ }^{\mathrm{a}, \mathrm{b}, \mathrm{c}^{*}}$ and Fereidoon Shahidid
}

\begin{abstract}
aSchool of Food Science and Technology, Dalian Polytechnic University, Dalian, PR China, 116034
${ }^{b}$ National Engineering Research Center of Seafood, Dalian, PR China, 116034

${ }^{c}$ Beijing Advanced Innovation Center of Food Nutrition and Human Health, China Agricultural University, Beijing, China, 100083

${ }^{\mathrm{d} D e p a r t m e n t}$ of Biochemistry, Memorial University of Newfoundland, St. John's, NL, Canada, A1B 3X9

*Corresponding author: Da-Yong Zhou, Bei-Wei Zhu, School of Food Science and Technology, Dalian Polytechnic University, Dalian, PR China, 116034.E-mail: zdyzf1@163.com, zhubeiwei@163.com
\end{abstract}

DOI: $10.31665 /$ JFB.2018.1132

Received: December 9, 2017; Revised received \& accepted: January 20, 2018

Citation: Zhou, X., Zhou, D.-Y., Yin, F.-W., Song, L., Liu, Y.-X., Xie, H.-K., Gang, K.-Q., Zhu, B.-W., and Shahidi, F. (2018). Glycerophospholipids in sea cucumber (Stichopus japonicus) and its processing by-products serve as bioactives and functional food ingredients. J. Food Bioact. 1: 134-142.

\begin{abstract}
Sea cucumber is a "healthy" food. Although previous studies have suggested that sea cucumber might serve as a potential rich source of polyunsaturated fatty acids (PUFAs) enriched phospholipid (PL), the molecular species of its PL has rarely been reported. In this study, some 200 glycerophospholipid (GP) species belonging to seven classes in processing by-products (spawns and intestines) of sea cucumber (Stichopus japonicus) were characterized for the first time. Most of the dominant GP species contained PUFAs, especially eicosapentaenoic acid (EPA, 20:5n-3) and arachidonic acid (AA, C20:4n-6). Meanwhile, the lipids contained high levels of PUFA (25.35-45.12\% of total FAs) and polar lipid (65.55-85.95\% of total lipids) but low levels of cholesterol (0.63-2.26\% of total lipids). Among PL, phosphatidylcholine (38.34-65.56 mol\%) was dominant. Therefore, PUFA enriched PL in sea cucumber may account for their nutritional and health beneficial effects. Meanwhile, by-products of Stichopus japonicus byproducts provide great potential as health-promoting food ingredients.
\end{abstract}

Keywords: Sea cucumber (Stichopus japonicus); processing by-products; lipid classes; phospholipid classes; molecular species; direct infusion mass spectrometry.

\section{Introduction}

Sea cucumber, an echinoderm, is one of the important aquatic species that is widely cultured and captured in China and many other Asian countries due to its high commercial value (Han et al., 2016). According to the 2015 statistics of Food and Agriculture Organization (FAO) of the United Nations, the total world production of sea cucumber was about 250 thousand tons (FAO, 2015). Sea cucumber is mainly consumed by Asian countries such as China, Korea, Japan, Vietnam, and Singapore as a delicacy, or a natural medicine (Han et al., 2016; Khotimchenko, 2015). During the processing of sea cucumber, internal organs, such as spawns and intestines, are discarded as byproducts. To reduce the burden on the environment and enhance their value, effective utilization of sea cucumber by-products is required.

Lipid is a type of important health-beneficial component in sea cucumber because of its abundant in health-beneficial polyunsaturated fatty acids (PUFAs) especially eicosapentaenoic acid (EPA, 20:5n-3) and arachidonic acid (AA, C20:4n-6) (Bechtel et al., 2013; Gao et al., 2016; Kasai, 2003). Meanwhile, lipids in 
sea cucumber tissues are also rich in phospholipids (PLs) (more than $40 \%$ of total lipids) (Drazen et al., 2008; Lou et al, 2012; Vaidya et al., 2014). A study by Lou et al. (2012) indicated that the phosphatidylcholine (PC) and phosphatidylethanolamine (PE) isolated from sea cucumber body wall contained a high percentage of PUFAs, in particular EPA and AA. Kostetsky et al. (2014) have characterized 25 species of glycerophosphocholine (GPCho) and 26 species of glycerophosphoethanolamine (GPEtn) from sea cucumber Apostichopus japonicus muscle tissues. Among them, the molecular species containing PUFA such as 20:4 and 20:5 were predominant.

Lately, the PL form of n-3 LC-PUFA have captured increasing consumer interest because PL are supposed to be highly effective in delivering their FA residues for incorporation into the membranes and altering the FA composition of membrane PLs within a certain cell type (Küllenberg et al., 2012). Therefore, oral supplementation of PL carrying n-3 PUFAs may contribute to higher health benefits of those who consume them. As described above, sea cucumber might serve as a potential rich source of PL enriched PUFA, and then provide great potential as health-promoting food ingredients. However, the fate of FA at $s n-1 / s n-2$ position of oral PL in vivo is different. In the intestinal lumen, dietary PL is cleaved into 1-lysophospholipid and FFA by activated pancreatic phospholipase $\mathrm{A}_{2}$ (Iqbal and Hussain, 2009; Phan and Tso, 2001). The resulting FFA is mainly re-synthesized into TAG whereas 1-lysophospholipid is mainly re-synthesized into PL before they are being transferred into the lymph and the blood (Cohn et al., 2008). Therefore, the determination of molecular species of GP may further reveal the heath-beneficial effects of PL-containing PUFA. So far, the GP molecular species in by-products of sea cucumber, as well as the GP molecular species other than GPCho and GPEtn in sea cucumber body wall have never been reported yet.

Sea cucumber Stichopus japonicus are the most common species widely cultured in China and Japan. Hence, the aim of this study was to determine the lipid content, lipid classes, PL classes, fatty acid composition and GP molecular species belong to seven classes including GPCho, GPEtn, glycerophosphoserine (GPSer), glycerophosphoinositol (GPIns), lysoglycerophosphocholine (LGPCho), lysoglycerophosphoethanolamine (LGPEtn) and lysoglycerophosphoserine (LGPSer) in body wall, spawns and intestines of sea cucumber Stichopus japonicus. This will help better understanding of health benefits of consumption of sea cucumber, as well as to provide theoretical basis for utilization of the processing by-products from sea cucumbers as novel sources of functional food ingredients.

\section{Materials and methods}

\subsection{Materials}

Sea cucumbers (Stichopus japonicus) were purchased from a local market in Dalian, Liaoning, China. Upon arrival, sea cucumbers were dissected and the body wall was collected for further experiment. Stichopus japonicus spawns and intestines were provided by Dalian Bangchuidao Seafood Co., LTD (Dalian, China). The samples were lyophilized in a freeze-dryer (2KBTES-55, VirTis Co., Gardiner, NY, USA) for $72 \mathrm{~h}$, crushed into powder and stored at $-30{ }^{\circ} \mathrm{C}$ until use. Deuterated chloroform $\left(\mathrm{CDCl}_{3}\right)$ and methanol (MeOD), triethyl phosphate (TEP), cesium carbonate $\left(\mathrm{CsCO}_{3}\right)$ and $\mathrm{D}_{2} \mathrm{O}$ were purchased from Aladdin Reagent Co., Ltd. (Shanghai, China). All other reagents were of analytical grade and purchased from Kemiou Chemical Reagent Co., Ltd. (Tianjin, China).

\subsection{Lipid extraction and sample preparation}

Total lipids were extracted from body wall, spawns and intestines of sea cucumber (Stichopus japonicus) by using the methyl tert-butyl ether (MTBE) method with slight modification (Matyash et al., 2008). First, $10 \mathrm{~g}$ powder sample were weighed and transferred to a $250 \mathrm{ml}$ conical flask. Then a mixture of methanol $(15 \mathrm{ml})$ and MTBE $(50 \mathrm{ml})$ was added to the conical flask. After being stirred for $60 \mathrm{~min}$ at $30^{\circ} \mathrm{C}, 12.5 \mathrm{ml}$ of deionized water was added to the mixture. Shaking the flask to fully mix the samples, then the mixture was transferred to centrifuge tub and centrifuged at 7,800 $\mathrm{g}$ for $10 \mathrm{~min}$ at 4 ${ }^{\circ} \mathrm{C}$. The organic layer was subsequently transferred to a glass sample bottle and the rest was re-extracted according to the aforementioned procedure. Finally, the organic layers were combined and concentrated to dryness with using a stream of nitrogen at $35^{\circ} \mathrm{C}$. The recovered lipids were stored at $-30^{\circ} \mathrm{C}$ for further analysis within 2 weeks.

\subsection{Determination of lipid class composition}

The determination of lipid classes was achieved by using an Iatroscan MK-6S thin layer chromatography-flame ionization detection (TLC-FID) Analyzer (Iatron Inc., Tokyo, Japan), according to Yin et al. (2015).

\subsection{Determination of fatty acid composition}

FA compositions were determined by using an Agilent 7890A GC-5975C MSD (Agilent, Palo Alto, CA,USA) equipped with an HP-5-MS capillary column (30 m, $0.25 \mathrm{~mm}, 0.25 \mu \mathrm{m})$ (Agilent), according to Yin et al. (2015).

\subsection{Quantitation of PL classes by ${ }^{31}$ P NMR}

The determination of PL classes was achieved by using an Avance III $400 \mathrm{MHz}$ NMR spectrometer (Bruker, Karlsruhe, Germany) (9.4 T), according to Liu et al. (2018).

\subsection{Mass spectrometer analysis of sea cucumber lipids}

In this study, qualitative and quantitative analyses of PLs were carried out on an hybrid API 4000 Qtrap (AB Sciex, Foster City, CA, USA) quadrupole-linear ion trap (QqLIT) mass spectrometer (MS) with a Turbo V electrospray ionization (ESI) sourceinterface, and a computer platform equipped with HPLC-MS/MS Solution Analystsoftware 1.6.1 (AB Sciex, Foster City, CA, USA). Sea cucumber lipids were dissolved in a mixture of methanol and chloroform $(1: 2, \mathrm{v} / \mathrm{v})$ with $0.1 \%$ of total solvent formic acid, filtered through a $0.22 \mu \mathrm{m}$ membrane, and directly introduced into the ESI source though a syringe pump. The sample concentration was $50 \mu \mathrm{g} / \mathrm{ml}$, and the flow rate was $5,15,15$ and $35 \mu \mathrm{l} / \mathrm{min}$, respectively, for analysis of GPCho/LGPCho, GPEtn/LGPEtn, GPSer/LGPSer and GPIns. In this study, precursor-ion scanning (PIS) and neutral loss scanning (NLS) were operated in the positive ion mode, while the enhanced product ion (EPI) scanning was operated in the negative ion mode. The instrument parameters were the same as used in our previous study (Liu et al., 2018).

A semi-quantitative method using an internal standard was used to compare the differences in the amount of the same GP molecular species between lipids recovered from different body parts of sea cucumber as previously described (Yin et al., 2016). In this study, 
Table 1. Fatty acid compositions (\%) of the lipids recovered from different body parts of sea cucumber (Stichopus japonicus)

\begin{tabular}{|c|c|c|c|}
\hline Fatty acid & 1 & 2 & 3 \\
\hline C14:1n-5 & $2.15 \pm 0.00^{a}$ & $0.35 \pm 0.03^{b}$ & $0.37 \pm 0.02^{b}$ \\
\hline C14:0 & $1.81 \pm 0.00^{\mathrm{a}}$ & $1.52 \pm 0.06^{b}$ & $0.50 \pm 0.03^{c}$ \\
\hline C15:0 & $3.77 \pm 0.01^{a}$ & $1.81 \pm 0.09^{b}$ & $1.31 \pm 0.07^{c}$ \\
\hline C16:1n-7 & $13.71 \pm 0.01^{a}$ & $9.52 \pm 0.41^{b}$ & $5.60 \pm 0.45^{c}$ \\
\hline $\mathrm{C} 16: 0$ & $16.18 \pm 0.10^{\mathrm{a}}$ & $6.78 \pm 0.33^{b}$ & $3.82 \pm 0.20^{c}$ \\
\hline C17:1n-7 & $1.24 \pm 0.02$ & nd & nd \\
\hline $\mathrm{C} 17: 0$ & $1.44 \pm 0.00^{b}$ & $2.11 \pm 0.09^{a}$ & $0.82 \pm 0.04^{c}$ \\
\hline$C 18: 4 n-3$ & $0.69 \pm 0.01$ & nd & nd \\
\hline C18:2n-6 & $4.08 \pm 0.01^{a}$ & $0.81 \pm 0.03^{c}$ & $1.56 \pm 0.15^{b}$ \\
\hline C18:1n-9 & $18.67 \pm 0.07^{a}$ & $11.01 \pm 0.10^{c}$ & $15.34 \pm 0.79^{b}$ \\
\hline C18:0 & $5.09 \pm 0.03^{b}$ & $9.16 \pm 0.18^{a}$ & $3.30 \pm 0.28^{c}$ \\
\hline C19:1n-9 & nd & $1.04 \pm 0.00$ & nd \\
\hline C19:0 & nd & $1.06 \pm 0.02^{a}$ & $0.64 \pm 0.01^{b}$ \\
\hline$C 20: 5 n-3$ & $8.57 \pm 0.03^{c}$ & $23.12 \pm 0.45^{a}$ & $22.16 \pm 0.48^{b}$ \\
\hline$C 20: 4 n-6$ & $8.64 \pm 0.07^{b}$ & $7.82 \pm 0.06^{c}$ & $11.97 \pm 0.22^{\mathrm{a}}$ \\
\hline$C 20: 3 n-6$ & nd & nd & $0.92 \pm 0.08$ \\
\hline C20:1n-9 & $7.64 \pm 0.00^{b}$ & $10.72 \pm 0.25^{a}$ & $10.64 \pm 0.89^{a}$ \\
\hline C20:0 & $1.12 \pm 0.00^{c}$ & $1.62 \pm 0.05^{a}$ & $1.49 \pm 0.04^{b}$ \\
\hline C21:0 & nd & nd & $1.09 \pm 0.02^{\mathrm{a}}$ \\
\hline$C 22: 6 n-3$ & $3.35 \pm 0.10^{c}$ & $4.35 \pm 0.04^{b}$ & $5.87 \pm 0.14^{\mathrm{a}}$ \\
\hline$C 22: 5 n-6$ & nd & $0.60 \pm 0.05^{b}$ & $0.98 \pm 0.08^{a}$ \\
\hline$C 22: 5 n-3$ & nd & $0.66 \pm 0.01^{b}$ & $1.06 \pm 0.02^{\mathrm{a}}$ \\
\hline$C 22: 4 n-6$ & nd & nd & $0.61 \pm 0.01$ \\
\hline$C 22: 1 n-9$ & $0.90 \pm 0.04^{c}$ & $3.48 \pm 0.19^{b}$ & $4.58 \pm 0.23^{a}$ \\
\hline $\mathrm{C} 22: 0$ & nd & $0.78 \pm 0.03^{b}$ & $1.17 \pm 0.03^{a}$ \\
\hline$C 24: 1 n-9$ & $0.93 \pm 0.08^{c}$ & $1.67 \pm 0.07^{b}$ & $4.34 \pm 0.24^{\mathrm{a}}$ \\
\hline SFA & $29.41 \pm 0.15^{a}$ & $24.85 \pm 0.36^{b}$ & $14.14 \pm 0.51^{c}$ \\
\hline MUFA & $45.24 \pm 0.03^{a}$ & $37.78 \pm 0.11^{c}$ & $40.86 \pm 0.51^{b}$ \\
\hline PUFA & $25.35 \pm 0.19^{c}$ & $37.36 \pm 0.47^{b}$ & $45.12 \pm 0.57^{a}$ \\
\hline
\end{tabular}

${ }^{a-c}$ Values in the same column with different lowercase letters are significantly different at $P<0.05$. Samples $1-3$ were lipids recovered from body wall, spawns and intestines of sea cucumber, respectively. Abbreviations are: SFA, saturated fatty acids; MUFA, monounsaturated fatty acids; PUFA, polyunsaturated fatty acids; and nd, not detected.

four GP standards including GPCho 12:0/12:0 (inner standard for GPCho/LGPCho), GPEtn 12:0/12:0 (inner standard for GPEtn/ LGPEtn), GPSer 12:0/12:0 (inner standard for GPSer/LGPSer) and GPIns 8:0/8:0 (inner standard for GPIns) were added to the samples as the internal standards to reach a concentration of $0.3,0.5,0.1$ and $0.1 \mu \mathrm{g} / \mathrm{ml}$, respectively, before injection for MS analysis.

\subsection{Statistical analysis}

The experiments were repeated for three times. Data were sub- jected to analysis with SPSS 16.0 software (SPSS Inc., Chicago, IL, USA). The results were denoted as mean \pm standard deviation (SD). Differences between means were evaluated by one-way analysis of variance (Student-Newman-Keuls post-hoc test) and independent sample T-test. $P$ values of $<0.05$ were considered statistically significant.

\section{Results and discussion}

\subsection{Lipid content}

The dried sample of body wall, spawns and intestines of sea cucumber (Stichopus japonicus) contained 6.83, 16.12 and 8.42\% lipids, respectively. Obviously, processing by-products (spawns and intestines) of Stichopus japonicus contained higher lipid contents than the body wall. Previous studies have also shown that the lipid contents of the gonads in male Stichopus japonicus were about twice of that in the body wall (Kasai, 2003).

\subsection{Fatty acid composition}

As shown in Table 1, unsaturated fatty acids (UFAs) such as palmitoleic acid (C16:1), oleic acid (C18:1), erucic acid (20:1), AA (C20:4 n-6), EPA (C20:5 n-3) and docosahexaenoic acid (DHA, $\mathrm{C} 22: 6 \mathrm{n}-3$ ) were dominant in lipids from all the three body parts of sea cucumber. Previous studies have also indicated that sea cucumber was abundant in the aforementioned FAs (Gao et al., 2016; Jing et al., 2010). However, their values varied dramatically with different body parts. PUFAs accounted for $25.35,37.36$ and $45.12 \%$ of total FAs of lipids, respectively, in the body wall, spawns and intestines. Obviously, by-products (intestines and spawns) of Stichopus japonicus contained higher PUFA contents but a lower content of saturated fatty acids (SFA) than the body wall. Similar results were previously reported by Zadorozhnyj et al., (2016) that PUFAs accounted for $37.55,41.92$ and $43.33 \%$ of total FAs, respectively, in the body wall, gonads and intestines of sea cucumber Apostichopus japonicus.

In this study, the PUFA, especially EPA $(22.16-23.12 \%$ of total FAs), DHA (4.35-5.87\% of total FAs) and AA (7.82-11.97\% of total FAs) were found to account for the majority of total FAs of by-products from Stichopus japonicus, indicating that the byproducts may serve as potential sources of EPA, DHA and AA. The omega-3 long-chain PUFA (n-3 LC-PUFA), in particular EPA and DHA, have received much attention in the past few decades because of their health benefits which include improving heart disease related outcomes, contributing to infant development, decreasing tumour growth and metastasis, inhibiting inflammation, platelet aggregation, hypertension, and hyperlipidaemia, and favourably modifying insulin sensitivity (Anderson and Ma, 2009; Riediger et al., 2009). Meanwhile, AA is indispensable for infant brain growth where it plays an important role in cell division and signaling (Katsuki and Okuda, 1995). Furthermore, it is noteworthy that the GP containing 20:4, 20:5 and 22:6 reported in this study are definitely AA, EPA and DHA as no other 20:4, 20:5 and 22:6 isomers are known to exist in sea cucumbers (Table 1).

\subsection{Lipid class composition}

The lipids from different body parts of Stichopus japonicus were composed of polar lipids, TAG, diacylglycerols (DAG), mono- 
Table 2. Lipid class compositions (\%) of the lipids recovered from different body parts of sea cucumber (Stichopus japonicus)

\begin{tabular}{lllllll}
\hline Samples & Polar Lipid & TAG & DAG & MAG & FFA & CHO \\
\hline 1 & $65.55 \pm 0.05^{\mathrm{c}}$ & $32.93 \pm 0.33^{\mathrm{a}}$ & $0.43 \pm 0.05^{\mathrm{a}}$ & $0.17 \pm 0.0^{\mathrm{b}}$ & nd & $0.63 \pm 0.00^{\mathrm{c}}$ \\
2 & $83.58 \pm 0.65^{\mathrm{b}}$ & $9.31 \pm 0.34^{\mathrm{b}}$ & $0.30 \pm 0.03^{\mathrm{b}}$ & $0.24 \pm 0.01^{\mathrm{a}}$ & $5.57 \pm 0.2^{\mathrm{b}}$ & $1.01 \pm 0.08^{\mathrm{b}}$ \\
3 & $85.95 \pm 0.46^{\mathrm{a}}$ & $5.37 \pm 0.16^{\mathrm{c}}$ & $0.24 \pm 0.08^{\mathrm{b}}$ & $0.30 \pm 0.05^{\mathrm{a}}$ & $5.97 \pm 0.16^{\mathrm{a}}$ & $2.26 \pm 0.26^{\mathrm{a}}$ \\
\hline
\end{tabular}

${ }^{a-c}$ Values in the same column with different lower-case letters are significantly different at $P<0.05$. Samples $1-3$ are lipids recovered from body wall, spawns and intestines of sea cucumber (Stichopus japonicus), respectively. Abbreviations are: TAG, triacylglycerol; DAG, diacylglycerol; MAG, monoacylglycerol; FFA, free fatty acid; and CHO, cholesterol.

acylglycerols (MAG), free fatty acids (FFA) and cholesterol (CHO) (Table 2). Obviously, polar lipids (65.55-85.95\% of total lipids) were dominant in the lipids of Stichopus japonicus. Lou et al. (2012) had previously reported that polar components including PLs $(43.32 \%$ of total lipids) and glycolipids $(23.82 \%$ of total lipids) were abundant in lipids from the body wall of Apostichopus japonicus. Meanwhile, Drazen et al. (2008) reported that PL accounted for $86.7,93.0,68.8$ and $95.2 \%$ of total lipids, respectively, in sea cucumber Abyssocucumis abyssorum, Oneirophanta mutabilis, Peniagone vitrea and Protankyra brychia. Furthermore, Stichopus japonicus lipids had impressively low CHO content $(0.63-2.26 \%$ of total lipids). Elevated total CHO levels are known to be one of the main risk factors for cardiovascular disease (Kennedy, 2016).

PLs constitute an important and widespread class of biomolecules of which GPs are of particular interest. They are the major components of all living cell membranes and have important structural and health-related benefits such as improved memory and immune function, prevention of heart disease and certain cancers (Matthan et al., 2014; Mürke et al., 2016; Nicolson and Ash, 2017). Our results showed that the by-products (spawns and intestines) of Stichopus japonicus contained higher PL but lower CHO content, indicating their potential application as functional food ingredients.

\subsection{Phospholipid class composition}

As shown in Table 3, PC, PE, phosphatidylserine (PS), phosphatidylinositol (PI), lysophosphatidylcholine (LPC), lysophosphatidylethanolamine (LPE), lysophosphatidylserine (LPS), phosphatidylglycerol (PG) and sphingomyelin (SM) were present in lipids from sea cucumber. Among them, PC (38.34-65.56 mol\%) was the primary lipid class, and PE (10.20-21.78 mol\%) and PS (8.56-11.68 mol\%) were secondary PL classes, while LPC (2.00$7.31 \mathrm{~mol} \%)$ and LPE (3.03-6.01 $\mathrm{mol} \%$ ) were present in small proportions. However, PI (8.23-14.87 mol\%), LPS (0.40-1.08 mol\%), PG (0.84-3.33 mol\%) and SM (1.74-4.18mol\%) were just observed in spawns and intestines. In contrast to body wall, the spawns and intestines of Stichopus japonicus contained more classes of PL, as well as a lower content in PC but higher contents in almost all other PL classes (except for LPC). Actually, previous studies have also shown that PC (40.4-75.4\% of the total PL), PE (11.5-34.2\% of the total PL), PS (3.6-18.3\% of the total PL) and PI (1.7-10.6\% of the total PL) were the top four PL classes in sea cucumber Cucumaria frondosa japonica, Eupentacta fraudatrix, and Apostichopus japonicus (Kostetsky et al., 2012).

Different types of PLs have varied nutritional value and health function. For example, PC is an essential part of human cell membrane and is important for healthy brain, heart, skeletal muscles, liver and metabolism (E Smith et al., 2016); PE is an abundant membrane PL that is essential for membrane integrity and cell division, and can be used to modify certain proteins (Calzada et al., 2016); PS, the major anionic PL class, is synthesized from PC or PE by exchanging the base head group with serine, and tends to improve nerve cell and brain memory function (Kim et al., 2014); PI is significant for a multiple of cellular functions including membrane trafficking, signal transduction, and cell growth (Picas et al., 2016). The lipids from the spawns and intestines of Stichopus japonicus have diverse and relatively balanced PL class profiles, indicating their special potential application as functional food ingredients.

\subsection{Characterization of glycerophospholipid molecular species}

Glycerophospholipids with different head groups can be selectively detected by PIS and NLS scanning modes using triple quadrupole mass spectrometry due to the loss of head groups from the molecules in MS/MS (Peterson and Cummings, 2006). In this study, a direct infusion MS/MS approach was used to characterize GP molecular species. PIS with $\mathrm{m} / \mathrm{z} 184$, and NLS with $\mathrm{m} / \mathrm{z} 141$, 185 , and 260 in the positive ion mode were used to selectively detect GPCho/LGPCho, GPEtn/LGPEtn, GPSer/LGPSer and GPIns, respectively. As shown in Figure 1, the candidate compounds for each class of the aforementioned GP showed visible first-stage MS signals in the PIS or NLS MS spectra. The measured $\mathrm{m} / \mathrm{z}$ value agreed with the molecular ion $\left([\mathrm{M}]^{+}\right)$of GPCho/LGPCho, and the quasi-molecular ion $\left([\mathrm{M}+\mathrm{H}]^{+}\right)$of GPEtn/LGPEtn, GPSer/LGPSer and GPIns, respectively. Therefore, the molecular mass of the unknown GP can be determined through the direct infusion MS/MS approach.

Common notation for a molecular species of GP follows the format $\mathrm{x}$ : $\mathrm{y}$ with $\mathrm{x}$ and $\mathrm{y}$ representing the number of carbons and

Table 3. Phospholipid class compositions (mol\%) of the lipids recovered from different body parts of sea cucumber (Stichopus japonicus)

\begin{tabular}{llllllllll}
\hline Sample & PC & PE & PS & PI & LPC & LPE & LPS & PG & SM \\
\hline 1 & $65.56 \pm 0.35^{\mathrm{a}}$ & $10.20 \pm 0.28^{\mathrm{c}}$ & $8.56 \pm 0.64^{\mathrm{c}}$ & $\mathrm{nd}$ & $7.31 \pm 0.67^{\mathrm{a}}$ & $3.03 \pm 0.95^{\mathrm{c}}$ & nd & nd & nd \\
2 & $41.05 \pm 0.54^{\mathrm{b}}$ & $21.78 \pm 1.00^{\mathrm{a}}$ & $10.26 \pm 0.48^{\mathrm{b}}$ & $8.23 \pm 0.62^{\mathrm{b}}$ & $5.22 \pm 0.43^{\mathrm{b}}$ & $6.01 \pm 0.26^{\mathrm{a}}$ & $0.40 \pm 0.11^{\mathrm{b}}$ & $0.84 \pm 0.13^{\mathrm{b}}$ & $4.18 \pm 0.24^{\mathrm{a}}$ \\
3 & $38.34 \pm 0.26^{\mathrm{c}}$ & $15.82 \pm 0.82^{\mathrm{b}}$ & $11.68 \pm 0.82^{\mathrm{a}}$ & $14.87 \pm 0.21^{\mathrm{a}}$ & $2.00 \pm 0.08^{\mathrm{c}}$ & $4.72 \pm 0.08^{\mathrm{b}}$ & $1.08 \pm 0.23^{\mathrm{a}}$ & $3.33 \pm 0.11^{\mathrm{a}}$ & $1.74 \pm 0.18^{\mathrm{b}}$ \\
\hline
\end{tabular}

${ }^{a-c}$ Values in the same column with different lower-case letters are significantly different at $P<0.05$. Samples $1-3$ are lipids recovered from body wall, spawns and intestines, respectively. Abbreviations are: PC, phosphatidylcholine; PE, phosphatidylethanolamine; PS, phosphatidylserine; PI, phosphatidylinositol; LPC, Iysophosphatidylcholine; LPE, lysophosphatidylethanolamine; LPS, lysophosphatidylserine; PG, phosphatidylglycerol; SM, sphingomyelin; and nd, not detected. 

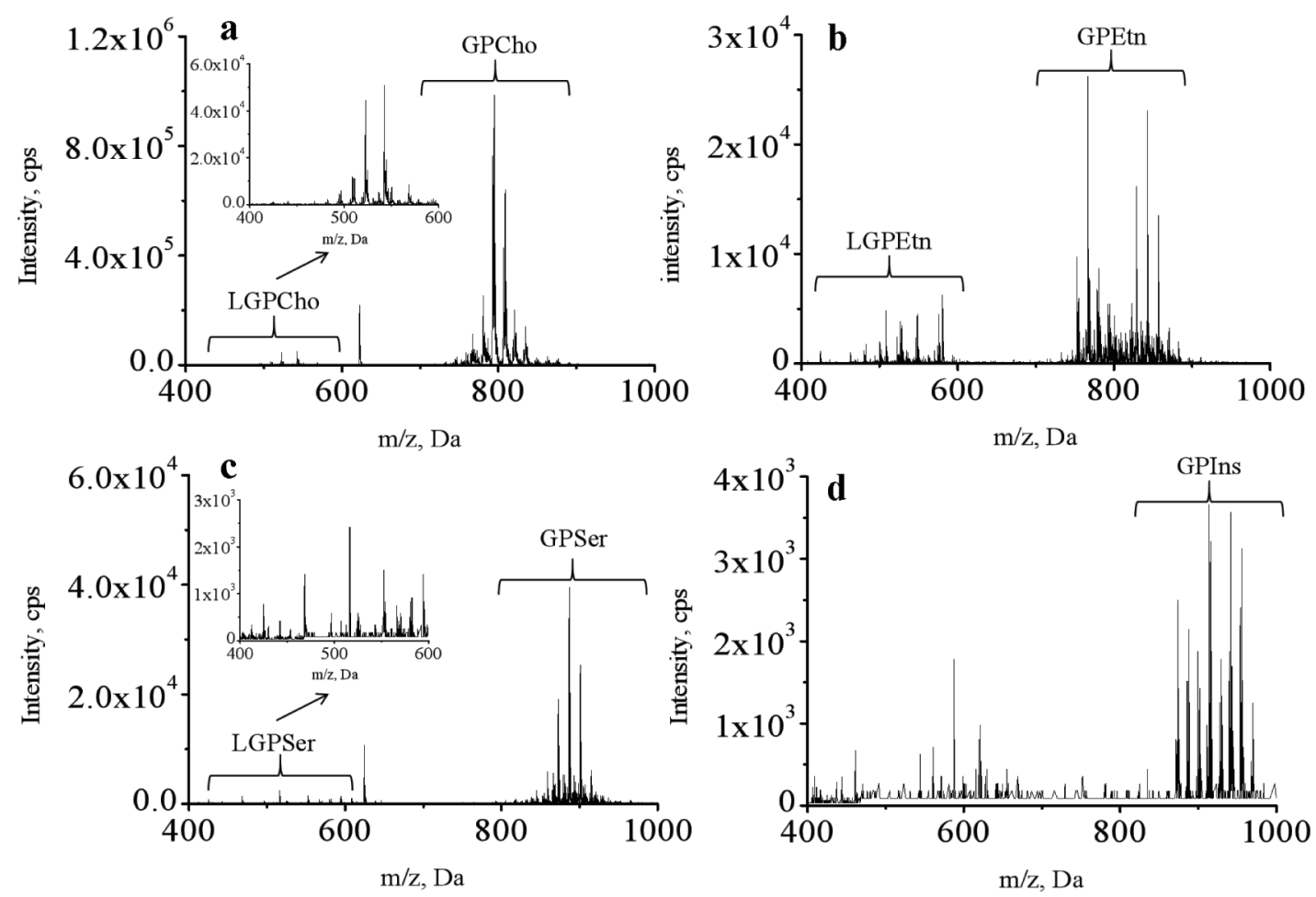

Figure 1. Specific detection of glycerophospholipids in lipids recovered from different body parts of sea cucumber by using direct infusion mass spectrometric approaches. a-d, first-stage MS spectra of glycerophosphocholines/lysoglycerophosphocholines (GPCho/LGPCho), glycerophosphoethanolamines/ lysoglycerophosphoethanolamines (GPEtn/LGPEtn), glycerophosphoserines/lysoglycerophosphoserines (GPSer/LGPSer) and glycerophosphatidylinositols (GPIns), respectively.

double bonds of FA in GP, respectively (Peterson et al., 2006). In our previous studies, formulas were developed to deduce the $\mathrm{x}$ and y of an unknown GP or lysoglycerophospholipid (LGP) based on its measured molecular mass (Liu et al., 2017; Yin et al., 2016). LGP contains just one FA, which means the measured molecular mass is sufficient for characterization of its molecular species. As shown in Tables 1S, the molecular species of detected LGP such as LGPCho in sea cucumber lipids were characterized according to their first stage MS data.

For an unknown GP containing two FAs, first stage MS data is insufficient for characterization of the two FAs which are esterified at the $s n-1$ and $s n-2$ positions of the glycerol backbone, therefore, MS/MS data are needed. In this study, EPI scanning in the negative ion mode was used to acquire the MS/MS data of the unknown GP. As shown in Tables 2S, one or even two FAs of an unknown GP such as GPCho can be characterized based on the MS/MS data. As described above, the total number of carbons and double bonds of the two FAs of an unknown GP can be tentatively deduced based on its first-stage MS data. Therefore, with the information for the total number of carbons and double bonds of the two FAs, and the structural information of one FA, the structural information for another FA of GP can be easily deduced. Furthermore, an empirical rule that the $s n-2$ of GP is the preferred position for the more unsaturated FAs was used to determine the distribution of the two FAs in the glycerol backbone (Napolitano et al., 1992). Through the above strategy, all detected GPCho, GPEtn, GPSer and GPIns were characterized based on their first-stage MS and MS/MS data.

Generally, approaches for structural characterization of plasmalogen (plasmenyl and plasmanyl) GP are more complex than that of non-plasmalogen GP. In most cases, the special FA fragments $\left([\mathrm{RCO}]^{-}\right)$of vinyl ether $\mathrm{x}:(\mathrm{y}-1)$ and alkyl ether $\mathrm{x}: \mathrm{y}$ which liberated from the $s n-1$ position of the plasmenyl and plasmanyl GP have the same $\mathrm{m} / \mathrm{z}$. This means a plasmenyl GP $\mathrm{x}:(\mathrm{y}-1)$ and a plasmanyl GP x:y may have the same first-stage MS and MS/MS data. Therefore, such a pair of possible structures could not be differentiated according to first-stage MS and MS/MS data. In this situation, a pair of possible structures including a plasmenyl GP $\mathrm{x}:(\mathrm{y}-1)$ and a plasmanyl GP x:y corresponding to the same first-stage MS and MS/MS data were counted as one GP.

\subsection{Glycerophospholipid molecular profile in different body parts of sea cucumber}

As shown in Tables 4 and 3S, at least 47 species of GPCho, 45, 44 and 46 species of GPEtn, 26 species of GPSer, as well as 17, 17 and 16 species of GPIns were characterized in lipids recovered from body wall, spawns and intestines of sea cucumber, respectively. Among them, 18:0/20:5, 18:1/20:4, 18:1/20:5, 22:0/20:5 were the predominant species of the phosphatidyl subclass, 18:0/20:4 and 22:0/22:1 might be the predominant species of the plasmenyl subclass, 18:0/20:5, 18:1/20:4, 20:0/20:5 and 22:1/22:1 might be the predominant species of the plasmanyl subclass (Table 5). Lou et al. (2012) reported that PC and PE from body wall of Apostichopus japonicus contained a large percentage of PUFA (57.60 and $44.99 \%$ of total FAs, respectively), particularly EPA $(27.90$ and $16.30 \%$ of total FAs, respectively) and AA (7.10 and $14.62 \%$ of total FAs, respectively). In this study, the by-products (spawns and intestines) of Stichopus japonicus had a higher level of PL 
Table 4. The number of species of glycerophosphocholines, glycerophosphoethanolamines, glycerophosphatidylserines, glycerophosphatidylinositols, lysoglycerophosphocholines, lysoglycerophosphoethanolamines and lysoglycerophosphatidylserines in lipids recovered from different body parts of sea cucumber (Stichopus japonicus)

\begin{tabular}{clll}
\hline Glycerophospholipid class & $\mathbf{1}$ & $\mathbf{2}$ & $\mathbf{3}$ \\
\hline Glycerophosphocholines & 47 & 47 & 47 \\
Phosphatidyl subclass & 14 & 14 & 14 \\
Plasmenyl or plasmanyl subclass & 33 & 33 & 33 \\
Glycerophosphoethanolamines & 45 & 44 & 46 \\
Phosphatidyl subclass & 26 & 24 & 26 \\
Plasmenyl or plasmanyl subclass & 19 & 20 & 20 \\
Glycerophosphatidylserines & 26 & 26 & 26 \\
Phosphatidyl subclass & 13 & 13 & 13 \\
Plasmenyl or plasmanyl subclass & 13 & 13 & 13 \\
Glycerophosphatidylinositol & 17 & 17 & 16 \\
Phosphatidyl subclass & 12 & 12 & 12 \\
Plasmenyl or plasmanyl subclass & 5 & 5 & 4 \\
Lysoglycerophosphocholines & 33 & 40 & 39 \\
Phosphatidyl subclass & 21 & 23 & 22 \\
Plasmenyl or plasmanyl subclass & 12 & 17 & 17 \\
Lysoglycerophosphoethanolamines & 20 & 23 & 23 \\
Phosphatidyl subclass & 12 & 14 & 14 \\
Plasmenyl or plasmanyl subclass & 8 & 9 & 9 \\
Lysoglycerophosphatidylserines & 7 & 13 & 10 \\
Phosphatidyl subclass & 6 & 8 & 8 \\
Plasmenyl or plasmanyl subclass & 1 & 5 & 2 \\
Total numbers & 195 & 210 & 207 \\
\hline
\end{tabular}

Samples 1-3 are lipids recovered from body wall, spawns and intestines of sea cucumber (Stichopus japonicus), respectively.

molecular species which contained 20:5 and 20:4 than body wall. Therefore, sea cucumber by-products might serve as a potential source of PL enriched EPA and AA.

Additionally, at least 33, 40 and 39 species of LGPCho, 20, 23 and 23 species of LGPEtn, and 7, 13 and 10 species of LGPSer were characterized for the first time in lipids recovered from body wall, spawns and intestines of sea cucumber, respectively (Table 4). Among them, 18:1, 20:1, 20:5 and 22:6 were the predominant species of the aforementioned PL classes (Table 5). By contrast, the by-product (spawns and intestines) of Stichopus japonicus had higher level of the LGPs molecular species than the body wall which contained 20:5 and 22:6.

In this study, some 200 species of GP belonging to classes of GPCho, GPEtn, GPSer, GPIns, LGPCho, LGPEtn and LGPSer were characterized in lipids recovered from different body parts of sea cucumber (Table 4). Previously, Kostetsky et al. (2014) characterized 25 species of GPCho and 26 species of GPEtn from muscle tissue of sea cucumbers. Among them, the molecular species containing 18:0/20:5 and 18:1/20:5 were abundant in GPCho, while 18:0/20:5 and 18:0/20:4 were predominant species of GPEtn, which are consistent with our results.
The PUFA in the PL form have recently captured increasing attention due to their have high bioavailability (Cook et al., 2016), high tissue-delivery capacity (Cansell, 2010; Rossmeisl et al., 2012), and good health promoting effects (Ramprasath et al., 2013; Ulven et al., 2011). Therefore, PUFA, especially EPA, AA and DHA in the PL form in Stichopus japonicus may account for much of its nutritional and healthy function. In particular, processing by-products of Stichopus japonicus might present a new source of PUFA in the PL form.

\section{Conclusion}

Freeze-dried body wall spawns and intestines of sea cucumber (Stichopus japonicus) contained 6.83, 16.12 and 8.42\% lipids, respectively. The lipids contained higher levels of PUFA (25.35$45.12 \%$ of total FAs), especially EPA (8.57-23.12\% of total FAs), DHA $(3.35-5.87 \%$ of total FAs) and AA $(7.82-11.97 \%$ of total FAs). These lipids were composed of polar lipid, TAG, DAG, MAG, FFA and $\mathrm{CHO}$, among which polar lipids $(65.55-85.95 \%$ of total lipids) were dominant while $\mathrm{CHO}(0.63-2.26 \%)$ were present in very small amounts. For PL, PC (38.34-65.56 mol\%) was the major component. Some 200 GP species were characterized in Stichopus japonicus body wall, spawns and intestines. Results indicated that most of the dominant molecular species of GP contained EPA and AA. Therefore, lipids in sea cucumber may account for their nutritional and health beneficial effects. Meanwhile, considering the high level of PUFA enriched GP but low level of $\mathrm{CHO}$, processing by-products (spawns and intestines) of sea cucumber Stichopus japonicus might have great potential as novel sources of health-promoting functional food ingredients.

\section{Acknowledgments}

This work was financially supported by "Public Science and Technology Research Funds Projects of Ocean (201505029)", "Project of Distinguished Professor of Liaoning Province (2015153)", "Program for Liaoning Excellent Talents in University (LR2015006), "Liaoning Provincial Natural Science Foundation of China (2015020781)", and Supported by Program for "Dalian High-Level Innovative Talent (2015R0007)".

\section{Supporting information}

Supplementary material for this article is available at https://doi. org/10.31665/JFB.2018.1132.

Table 1S. Lysoglycerophosphocholines in lipids recovered from different body parts of sea cucumber (Stichopus japonicus). The ratio of the intensity of the first-stage MS signal of a lysoglycerophosphocholine in the positive ion mode to that of the corresponding internal standard was used to represent its relative amount for comparison.

Table 2S. Structural identification of glycerophosphocholines in lipids recovered from different body parts of sea cucumber (Stichopus japonicus) according to first-stage MS and MS/MS data.

Table 3S. Glycerophosphocholines in lipids recovered from different body parts of sea cucumber (Stichopus japonicus). The ratio of the intensity of the first-stage MS signal of a glycerophos- 
Table 5. Major glycerophosphocholines, glycerophosphoethanolamines, glycerophosphatidylserines, glycerophosphatidylinositols, lysoglycerophosphocholines, lysoglycerophosphoethanolamines and lysoglycerophosphatidylserines in lipids recovered from different body parts of sea cucumber (Stichopus japonicus)

\begin{tabular}{|c|c|c|c|c|c|}
\hline \multirow{2}{*}{ Classes } & \multirow{2}{*}{$\begin{array}{l}\text { Measured } \mathrm{m} / \mathrm{z} \\
([\mathrm{M}]+) \text { or }([\mathrm{M}+\mathrm{H}]+)^{d}\end{array}$} & \multirow{2}{*}{ Molecular species } & \multicolumn{3}{|c|}{ Relative intensity } \\
\hline & & & Body & wall & Spawn \\
\hline \multirow[t]{12}{*}{ GPCho } & 794 & Plasmanyl 18:0/20:5 & $1.93 \pm 0.15^{c}$ & $4.18 \pm 0.14^{a}$ & $2.45 \pm 0.23^{b}$ \\
\hline & & Plasmanyl 18:1/20:4 & & & \\
\hline & & Plasmenyl 18:0/20:4 & & & \\
\hline & & Plasmanyl 18:4/20:1 & & & \\
\hline & & Plasmenyl 18:3/20:1 & & & \\
\hline & 796 & Plasmanyl 18:3/20:1 & $1.06 \pm 0.10^{b}$ & $1.47 \pm 0.08^{\mathrm{a}}$ & $0.75 \pm 0.05^{c}$ \\
\hline & & Plasmenyl 18:2/20:1 & & & \\
\hline & & Plasmanyl 20:3/18:1 & & & \\
\hline & & Plasmenyl 20:2/18:1 & & & \\
\hline & 806 & Phosphatidyl 18:1/20:5 & $0.62 \pm 0.03^{c}$ & $1.83 \pm 0.03^{a}$ & $1.39 \pm 0.03^{b}$ \\
\hline & 808 & Phosphatidyl 18:0/20:5 & $0.99 \pm 0.04^{c}$ & $2.80 \pm 0.03^{a}$ & $1.08 \pm 0.03^{b}$ \\
\hline & 810 & Phosphatidyl 18:3/20:1 & $0.69 \pm 0.00^{b}$ & $1.24 \pm 0.02^{\mathrm{a}}$ & $0.48 \pm 0.07^{c}$ \\
\hline \multirow[t]{8}{*}{ GPEtn } & 752 & Plasmanyl 18:1/20:4 & $0.16 \pm 0.01^{c}$ & $2.40 \pm 0.20^{a}$ & $0.52 \pm 0.06^{b}$ \\
\hline & & Plasmenyl 18:0/20:4 & & & \\
\hline & 766 & Phosphatidyl 18:0/20:5 & $0.25 \pm 0.03^{c}$ & $6.43 \pm 0.34^{a}$ & $0.74 \pm 0.03^{b}$ \\
\hline & & Phosphatidyl 18:1/20:4 & & & \\
\hline & 780 & Plasmanyl 20:0/20:5 & $0.15 \pm 0.02^{c}$ & $2.02 \pm 0.13^{a}$ & $0.50 \pm 0.05^{b}$ \\
\hline & 828 & Phosphatidyl 20:1/22:1 & $0.17 \pm 0.03^{b}$ & $2.98 \pm 0.39^{a}$ & $0.35 \pm 0.03^{b}$ \\
\hline & 842 & Plasmanyl 22:1/22:1 & $0.25 \pm 0.01^{c}$ & $3.42 \pm 0.11^{\mathrm{a}}$ & $0.49 \pm 0.04^{b}$ \\
\hline & & Plasmenyl 22:0/22:1 & & & \\
\hline \multirow[t]{11}{*}{ GPSer } & 872 & Phosphatidyl 22:1/20:1 & $0.55 \pm 0.12^{c}$ & $2.39 \pm 0.24^{a}$ & $1.92 \pm 0.05^{b}$ \\
\hline & 886 & Plasmanyl 24:1/20:1 & $1.31 \pm 0.12^{b}$ & $3.36 \pm 0.35^{a}$ & $3.66 \pm 0.06^{a}$ \\
\hline & & Plasmenyl 24:0/20:1 & & & \\
\hline & & Plasmanyl 22:1/22:1 & & & \\
\hline & & Plasmenyl 22:0/22:1 & & & \\
\hline & 888 & Plasmanyl 24:1/20:0 & $0.57 \pm 0.07^{b}$ & $1.59 \pm 0.22^{\mathrm{a}}$ & $1.33 \pm 0.15^{a}$ \\
\hline & & Plasmenyl 24:0/20:0 & & & \\
\hline & & Plasmanyl 24:0/20:1 & & & \\
\hline & 900 & Phosphatidyl 20:1/24:1 & $0.71 \pm 0.07^{b}$ & $2.22 \pm 0.09^{a}$ & $2.33 \pm 0.17^{a}$ \\
\hline & & Phosphatidyl 22:1/22:1 & & & \\
\hline & 902 & Phosphatidyl 20:0/24:1 & $20: 0 / 24: 1$ & $0.38 \pm 0.03^{b}$ & $0.89 \pm 0.05^{a}$ \\
\hline \multirow[t]{7}{*}{ GPIns } & 913 & Phosphatidyl 18:3/22:2 & $1.48 \pm 0.24^{a}$ & $1.31 \pm 0.23^{a}$ & $0.45 \pm 0.03^{b}$ \\
\hline & & Phosphatidyl 20:1/20:4 & & & \\
\hline & 915 & Phosphatidyl 20:0/20:4 & $1.54 \pm 0.10^{a}$ & $1.65 \pm 0.04^{a}$ & $0.59 \pm 0.01^{b}$ \\
\hline & 941 & Phosphatidyl 22:1/20:4 & $1.26 \pm 0.09^{b}$ & $2.23 \pm 0.24^{\mathrm{a}}$ & $0.76 \pm 0.00^{c}$ \\
\hline & 943 & Phosphatidyl 22:0/20:4 & $0.67 \pm 0.06^{a}$ & $0.82 \pm 0.08^{a}$ & $0.33 \pm 0.08^{c}$ \\
\hline & 955 & Plasmanyl 24:1/20:4 & $1.12 \pm 0.12^{b}$ & $1.37 \pm 0.05^{a}$ & $0.68 \pm 0.10^{c}$ \\
\hline & & Plasmenyl 24:0/20:4 & & & \\
\hline LGPCho & 508.7 & Plasmanyl 18:1 & $0.0122 \pm 0.0005^{c}$ & $0.0432 \pm 0.0029^{b}$ & $0.2180 \pm 0.0338^{a}$ \\
\hline
\end{tabular}


Table 5. Major glycerophosphocholines, glycerophosphoethanolamines, glycerophosphatidylserines, glycerophosphatidylinositols, lysoglycerophosphocholines, lysoglycerophosphoethanolamines and lysoglycerophosphatidylserines in lipids recovered from different body parts of sea cucumber (Stichopus japonicus)

\begin{tabular}{|c|c|c|c|c|c|}
\hline \multirow{2}{*}{ Classes } & \multirow{2}{*}{$\begin{array}{l}\text { Measured } \mathrm{m} / \mathrm{z} \\
([\mathrm{M}]+) \text { or }([\mathrm{M}+\mathrm{H}]+)^{d}\end{array}$} & \multirow{2}{*}{ Molecular species } & \multicolumn{3}{|c|}{ Relative intensity } \\
\hline & & & Body & wall & Spawn \\
\hline & & Plasmenyl 18:0 & & & \\
\hline & 522.6 & Phosphatidyl 18:1 & $0.0291 \pm 0.0022^{c}$ & $0.1666 \pm 0.0063^{b}$ & $0.2068 \pm 0.0162^{a}$ \\
\hline & 542.4 & Phosphatidyl 20:5 & $0.0172 \pm 0.0008^{c}$ & $0.1966 \pm 0.0052^{a}$ & $0.1209 \pm 0.0049^{b}$ \\
\hline & 568.2 & Phosphatidyl 22:6 & $0.0170 \pm 0.0022^{c}$ & $0.0354 \pm 0.0010^{b}$ & $0.0783 \pm 0.0063^{a}$ \\
\hline \multirow[t]{5}{*}{ LGPEtn } & 508.4 & Phosphatidyl 20:1 & $0.1530 \pm 0.0050^{c}$ & $0.6368 \pm 0.0162^{a}$ & $0.2047 \pm 0.0442^{b}$ \\
\hline & 522.6 & Plasmanyl 22:1 & $0.0531 \pm 0.0051^{c}$ & $0.3480 \pm 0.0353^{a}$ & $0.2369 \pm 0.0119^{b}$ \\
\hline & Plasmenyl 22:0 & & & & \\
\hline & 526.6 & Phosphatidyl 22:6 & $0.0736 \pm 0.0049^{c}$ & $0.6951 \pm 0.0532^{\mathrm{a}}$ & $0.2693 \pm 0.0273^{b}$ \\
\hline & 528.4 & Phosphatidyl 22:5 & $0.0543 \pm 0.0039^{c}$ & $0.6101 \pm 0.0177^{a}$ & $0.2271 \pm 0.0096^{b}$ \\
\hline \multirow[t]{4}{*}{ LGPSer } & 468.4 & Phosphatidyl 14:1 & $0.1678 \pm 0.0135^{b}$ & $0.3636 \pm 0.1427^{a}$ & $0.1562 \pm 0.0443^{c}$ \\
\hline & 524.8 & Phosphatidyl 18:1 & $18: 1$ & $0.0328 \pm 0.0035^{c}$ & $0.0424 \pm 0.0132^{b}$ \\
\hline & 526.4 & Phosphatidyl 18:0 & 18:0 & $0.0443 \pm 0.0031^{c}$ & $0.0528 \pm 0.0050^{b}$ \\
\hline & 552.4 & Phosphatidyl 20:1 & $20: 1$ & $0.0446 \pm 0.0074^{c}$ & $0.1154 \pm 0.0207^{b}$ \\
\hline
\end{tabular}

${ }^{a-c}$ Values in the same line with different lower-case letters are significantly different at $P<0.05 .{ }^{d}[\mathrm{M}]^{+}$for glycerophosphocholines and lysoglycerophosphocholines; $[\mathrm{M}+\mathrm{H}]^{+}$for glycerophosphoethanolamines, lysoglycerophosphoethanolamines, glycerophosphatidylserines, lysoglycerophosphatidylserines and glycerophosphatidylinositols. Abbreviations are: GPCho, glycerophosphocholine; GPEtn, glycerophosphoethanolamine; GPSer, glycerophosphatidylserine; GPIns, glycerophosphatidylinositol; LGPCho, lysoglycerophosphocholine; LGPEtn, lysoglycerophosphoethanolamine; and LGPSer, lysoglycerophosphatidylserine. The ratio of the intensity of the first-stage MS signal of a glycerophospholipid in the positive ion mode to that of the corresponding internal standard represents its relative amount for comparison.

phocholine in the positive ion mode to that of the corresponding internal standard was used to represent its relative amount for comparison.

\section{References}

Anderson, B.M., and Ma, D.W.L. (2009). Are all n-3 polyunsaturated fatty acids created equal? Lipids Health Dis 8: 1-20.

Bechtel, P.J., Oliveira, A., Demir, N., and Smiley, S. (2013). Chemical composition of the giant red sea cucumber, Parastichopus californicus, commercially harvested in Alaska. Food Sci Nutr 1: 63-73.

Calzada, E., Onguka, O., and Claypool, S.M. (2016). Phosphatidylethanolamine metabolism in health and disease. Int Rev Cell Mol Biol 321: 29-88.

Cansell, M. (2010). Marine phospholipids as dietary carriers of long-chain polyunsaturated fatty acids. Lipid Technology 22: 223-226.

Cohn, J.S., Wat, E., Kamili, A., and Tandy, S. (2008). Dietary phospholipids, hepatic lipid metabolism and cardiovascular disease. Curr Opin Lipidol 19: 257-262.

Cook, C.M., Hallaråker, H., Sæbø, P.C., Innis, S.M., Kelley, K.M., Sanoshy, K.D., and Maki, K.C. (2016). Bioavailability of long chain omega-3 polyunsaturated fatty acids from phospholipid-rich herring roe oi in men and women with mildly elevated triacylglycerols. Prostaglandins Leukot Essent Fatty Acids 111: 17-24.

Drazen, J.C., Phleger, C.F., Guest, M.A., and Nichols, P.D. (2008). Lipid, sterols and fatty acid composition of abyssal holothurians and ophiuroids from the North-East Pacific Ocean: Food web implications. Comp Biochem Physiol B Biochem Mol Biol 151: 79-87.

E Smith, R., Rouchotas, P., and Fritz, H. (2016). Lecithin (Phosphatidylcholine): Healthy Dietary Supplement or Dangerous Toxin? J Nat Prod 6 : 242-249.

FAO. (2015). FIGIS list of species for fishery global production statistics. URL http://www.fao.org/figis/servlet/TabSelector.

Gao, Y., Li, Z., Qi, Y., Guo, Z., Lin, Y., Li, W., Hu, Y.C., and Zhao, Q.C. (2016).
Proximate composition and nutritional quality of deep sea growth sea cucumbers (stichopus japonicus) from different origins. J Sci Food Agric 96: 2378-2383.

Han, Q., Keesing, J.K., and Liu, D. (2016). A review of sea cucumber aquaculture, ranching, and stock enhancement in china. Rev Fish Sci Aquac 24: 326-341.

Iqbal, J., and Hussain, M.M. (2009). Intestinal lipid absorption. Am J Physiol Endocrinol Metab 296: E1183-E1194.

Jing, W., Hu, C., and Fan, S. (2010). Chemical composition and nutritional quality of sea cucumbers. J Sci Food Agric 90: 2469-2474.

Kasai, T. (2003). Lipid contents and fatty acid composition of total lipid of sea cucumber stichopus japonicus and konowata (salted sea cucumber entrails). Food Sci Technol Res 9: 45-48.

Katsuki, H., and Okuda, S. (1995). Arachidonic acid as a neurotoxic and neurotrophic substance. Prog Neurobiol 46: 607-636.

Kennedy, E. (2016). Diet and cholesterol; a review of the current evidence. Nursing in General Practice 12-14.

Kim, H.Y., Huang, B.X., and Spector, A.A. (2014). Phosphatidylserine in the brain: metabolism and function. Prog Lipid Res 56: 1-18.

Khotimchenko, Y.S. (2015). The nutritional value of holothurians. Russ Mar Biol 41: 409-423.

Kostetsky, E.Y., Sanina, N.M., and Velansky, P.V. (2014). The thermotropic behavior and major molecular species composition of the phospholipids of echinoderms. Russ J Mar Biol 40: 131-139.

Kostetsky, E.Y., Velansky, P.V., and Sanina, N.M. (2012). Phospholipids of the organs and tissues of echinoderms and tunicates from peter the great bay (Sea of Japan). Russ J Mar Biol 38: 64-71.

Küllenberg, D., Taylor, L.A., Schneider, M., and Massing, U. (2012). Health effects of dietary phospholipids. Lipids Health Dis 11: 3.

Liu, Z.Y., Zhou, D.Y., Wu, Z.X., Yin, F.W., Zhao, Q., Xie, H.K., Zhang, J.R., Qin L., and Shahidi, F. (2018). Extraction and detailed characterization of phospholipid- enriched oils from six species of edible clams. Food Chem 239: 1175-1181.

Liu, Z.Y., Zhou, D.Y., Zhao, Q., Yin, F.W., Hu, X.P., Song, L., Qin, L., Zhang, J.R., Zhu, B.W., and Shahidi, F. (2017). Characterization of glycerophospholipid molecular species in six species of edible clams by high- 
performance liquid chromatography-electrospray ionization-tandem mass spectrometry. Food Chem 219: 419-427.

Lou, Q.M., Wang, Y.M., Liu, X.F., and Xue, C.H. (2012). Lipid profile and fatty acid compositions in body wall of Apostichopus Japonicus (Selenka). J Food Biochem 36: 317-321.

Matthan, N.R., Ooi, E.M., Van Horn, L., Neuhouser, M.L., Woodman, R., and Lichtenstein, A.H. (2014). Plasma Phospholipid Fatty Acid Biomarkers of Dietary Fat Quality and Endogenous Metabolism Predict Coronary Heart Disease Risk: A Nested Case-Control Study Within the Women's Health Initiative Observational Study. J Am Heart Assoc 3: e000764.

Matyash, V., Liebisch, G., Kurzchalia, T.V., Shevchenko, A., and Schwudke, D. (2008). Lipid extraction by methyl-tert-butyl ether for highthroughput lipidomic. J Lipid Res 49: 1137-1146.

Mürke, E., Stoll, S., Lendeckel, U., Reinhold, D., and Schild, L. (2016). The mitochondrial phospholipid cardiolipin is involved in the regulation of T-cell proliferation. Biochim Biophys Acta 1861: 748-754.

Napolitano, G.E., MacDonald, B.A., Thompson, R.J., and Ackman, R.G. (1992). Lipid composition of eggs and adductor muscle in giant scallops (Placopecten magellanicus) from different habitats. Marine Biology 113: 71-76.

Nicolson, G.L., and Ash, M.E. (2017). Membrane Lipid Replacement for chronic illnesses, aging and cancer using oral glycerolphospholipid formulations with fructooligosaccharides to restore phospholipid function in cellular membranes, organelles, cells and tissues. Biochim Biophys Acta 9: 1704-1724.

Peterson, B.L., and Cummings, B.S. (2006). A review of chromatographic methods for the assessment of phospholipids in biological samples. Biomed Chromatogr 20: 227-243.

Picas, L., Gaits-lacovoni, F., and Goud, B. (2016). The emerging role of phosphoinositide clustering in intracellular trafficking and signal transduction. F1000Res 5: 442.

Ramprasath, V.R., Eyal, I., Zchut, S., and Jones, P.J. (2013). Enhanced in- crease of omega-3 index in healthy individuals with response to 4-week n-3 fatty acid supplementation from krill oil versus fish oil. Lipids Health Dis 12: 178

Phan, C.T., and Tso, P. (2001). Intestinal lipid absorption and transport. Front Biosci 6: D299-319.

Riediger, N.D., Othman, R.A., Suh, M., and Moghadasian, M.H. (2009). A systemic review of the roles of n-3 fatty acids in health and disease. J Am Diet Assoc 109: 668-679.

Rossmeisl, M., Jilkova, Z.M., Kuda, O., Jelenik, T., Medrikova, D., Stankova, B., Kristinsson, B., Haraldsson, G.G., Svensen, H., Stoknes, I., Sjövall, P., Magnusson, Y., Balvers, M.G., Verhoeckx, K.C., Tvrzicka, E., Bryhn, M., and Kopecky, J. (2012). Metabolic effects of n-3 PUFA as phospholipids are superior to triglycerides in mice fed a high-fat diet: possible role of endocannabinoids. PloS One 7: e38834.

Ulven, S.M., Kirkhus, B., Lamglait, A., Basu, S., Elind, E., Haider, T., Berge, K., Vik, H., and Pedersen, J.I. (2011). Metabolic effects of krill oil are essentially similar to those of fish oil but at lower dose of EPA and $\mathrm{DHA}$, in healthy volunteers. Lipids 46: 37-46.

Vaidya, H., and Cheema, S.K. (2014). Sea cucumber and blue mussel: new sources of phospholipid enriched omega-3 fatty acids with a potential role in 3t3-I1 adipocyte metabolism. Food Funct 5: 3287-3295.

Yin, F.W., Liu, X.Y., Fan, X.R., Zhou, D.Y., Xu, W.S., Zhu, B.W., and Murata, Y. (2015). Extrusion of Antarctic krill (Euphausia superba) meal and its effect on oil extraction. Int J Food Sci Tech 50: 633-639.

Yin, F.W., Zhou, D.Y., Zhao, Q., Liu, Z.Y., Hu, X.P., Liu, Y.F., Song, L., Zhou, X., Zhu, B.W., and Shahidi, F. (2016). Identification of glycerophospholipid molecular species of mussel (Mytilus edulis) lipids by highperformance liquid chromatography-electrospray ionization-tandem mass spectrometry. Food Chem 213: 344-351.

Zadorozhnyj, P.A., Pivnenko, T.N., and Kovalev, N.N. (2016). Development of fatty acid biomarkers for the identification of wild and aquacultured sea cucumber (Apostichopus japonicus). J Ocean Univ China 15: 177-183. 